\title{
Pilot Cyclic Prefixed Single Carrier Communication: Channel Estimation and Equalization
}

\author{
Yonghong Zeng, Member, IEEE, and Tung Sang Ng, Fellow, IEEE
}

\begin{abstract}
Cyclic prefixed single carrier (CP-SC) has emerged as a promising technique for wideband wireless communication. The cyclic prefix (CP) in CP-SC costs some bandwidth, but it cannot be used for channel estimation in fast-variant channel environment. In this letter, a new scheme is proposed for CP-SC which not only adds a CP but also a suffix. With the aid of the CP and suffix, channel responses can be resolved by the fast Fourier transform. The additional known symbols also ensure input symbols recoverable regardless of the channel null locations. Better synchronization is also possible.
\end{abstract}

Index Terms-Channel estimation, CP-SC, cyclic prefix, equalization, fast fading, single carrier.

\section{INTRODUCTION}

C YCLIC PREFIXED single carrier (CP-SC) communication [1]-[5], which is also called single carrier with frequency-domain equalization (SC-FDE), has nearly the same advantages of orthogonal frequency division multiplexing (OFDM), but avoids OFDM's shortcomings of high peak-to-average power ratio (PAPR) and high sensitivity to frequency errors. Therefore, $\mathrm{CP}-\mathrm{SC}$ has emerged as a promising technique for wideband wireless communication. In both CP-SC and OFDM, a cyclic prefix (CP) is used to cope with time dispersive channels. For pseudo-static channel environment, the CP may be helpful for channel estimation in some blind or adaptive methods as shown in [6]-[9] for OFDM. However, it is barely useful for channel estimation in fast-variant channel environment, because blind or adaptive method needs relatively large number of stationary output samples to compute statistical values (for example, correlation matrix [6], [7], high-order statistics of outputs [9]) or to achieve convergence [8]. Another problem in OFDM and CP-SC is that symbols are theoretically not recoverable when some channel nulls meet the transmitted subcarriers or the discrete Fourier transform (DFT) of channel has zero (or near) coefficients.

In [2], a modified CP-SC called training aided single carrier (TASC) is proposed, which replaces the CP by a known constant data block. This approach is also discussed in [3], [4]. TASC does not need more bandwidth than CP-SC, and achieves

Manuscript received May 4, 2004; revised July 7, 2004. This work was supported by Grant HKU 7164/04E from the Research Grants Council of the Hong Kong SAR, China. The associate editor coordinating the review of this manuscript and approving it for publication was Prof. Jonathon A. Chambers.

Y. Zeng was with the Department of Electrical and Electronic Engineering, The University of Hong Kong, Hong Kong. He is now with the Institute for Infocomm Research, A-STAR, Singapore 119613 (e-mail: yhzeng@ ieee.org).

T. S. Ng is with the Department of Electrical and Electronic Engineering, The University of Hong Kong, Hong Kong (e-mail: tsng@eee.hku.hk).

Digital Object Identifier 10.1109/LSP.2004.839698 $v(0) v(1) \cdots v(L-1) s_{i}(0) s_{i}(1) \cdots s_{i}\left(N_{1}-1\right) v(0) v(1) \cdots v(L-1)$

Fig. 1. Transmitting block structure.

synchronization by using the $\mathrm{CP}$ as a training sequence. However, it is still unable to estimate the channel by using only the known data block [2]. In this letter, we propose a pilot cyclic prefixed SC (PCP-SC) which has the ability to achieve channel estimation in fast-fading environment. The PCP-SC modifies the CP-SC by adding a known constant block as a suffix to each symbol block before performing the cyclic prefixing. The structure offers a simple channel estimation by FFT. The compromise is that the data rate is somewhat decreased. It can also be viewed as doubling the size of the known data block in TASC and therefore leads to enhancement in synchronization. The additional known symbols also enable symbol recoverable even if the DFT of the channel has nulls. New equalization methods are also proposed, which are shown to achieve better performance.

In the following, superscripts $T$, $\dagger$, and $*$ stand for transpose, Hermitian (trans-conjugate), and complex conjugate, respectively, and I stands for an identity matrix.

\section{System StRUCTURE AND CHANNEL ESTIMATION}

At the transmitting end, the PCP-SC differs from the original CP-SC [1], [3]-[5] only in the block structure. Unlike CP-SC, the PCP-SC first adds a known block as a suffix to each symbol block and then performs the cyclic prefixing. Since the CP is now a known symbol block, it can be used as pilot. The block structure is shown in Fig. 1. Let $s_{i}(n)$ be the $n$th symbol in the $i$ th block to be transmitted, where $i=0,1, \cdots$; $n=0,1, \cdots, N_{1}-1$. Each block is padded with a known length- $L$ block $v(n), n=0,1, \cdots, L-1$, to the tail, where $v(n)$ is fixed (constant) for all blocks. Therefore, the expanded block is of length $N=N_{1}+L$, which is denoted by $\bar{s}_{i}(n)$, $n=0,1, \cdots, N-1$. As in the conventional CP-SC, a CP of length $L$ is added to each expanded block. Hence, the actual block to be transmitted is of length $M=N+L$. We use $\hat{s}_{i}(n)$ to denote such a block, where $n=0,1, \cdots, M-1$.

At the receiving end, time/frequency synchronization must be done before channel estimation. In each transmitted block, there are $2 L$ known elements. Based on this, joint time/frequency synchronization can be done, which is discussed in detail in [2]. Here, we focus on channel estimation. The received $i$ th block is denoted by $x_{i}(k)(k=0,1, \cdots, M-1)$. Instead of discarding the first $L$ elements as in the conventional single carrier system, these $L$ elements are used for channel estimation as follows. 
Theorem 1: Assume that the multipath channel is invariant within one block, and its delay spread is less than $L T$, where $T$ is the symbol interval. Let $h_{i}(l)(l=0,1, \cdots, L-1)$ denote the discrete channel filter in the $i$ th block. Then, $x_{i}(k)$ $(k=0,1, \cdots, L-1)$ is the cyclic convolution of $v(n)(n=$ $0,1, \cdots, L-1)$ and $h_{i}(l)(l=0,1, \cdots, L-1)$ plus the noise, that is

$x_{i}(k)=\sum_{l=0}^{L-1} h_{i}(l) v\left(\langle k-l\rangle_{L}\right)+\eta_{i}(k), \quad k=0,1, \cdots, L-1$

where $\langle k-l\rangle_{L}$ is the least nonnegative residue of $k-l$ modulo $L$, and $\eta_{i}(k)$ is the noise.

Proof: The received signal is the convolution of the channel and transmitted signal. Since the length of the channel is $L$, the first $L-1$ elements of $x_{i}(k)$ not only depend on the $i$ th symbol block but also $(i-1)$ th symbol block, that is, there exists inter-block interference (IBI). In general, we have

$x_{i}(k)=\sum_{l=0}^{k} h_{i}(l) \hat{s}_{i}(k-l)+\sum_{l=k+1}^{L-1} h_{i}(l) \hat{s}_{i-1}(M+k-l)+\eta_{i}(k)$,

$$
k=0,1, \cdots, L-1 \text {. }
$$

Since the last $L$ elements of block $(i-1)$ is $v$, that is, $\hat{s}_{i-1}(n+$ $M-L)=v(n)(n=0,1, \cdots, L-1)$, we have $\hat{s}_{i-1}(M+k-$ $l)=v(L+k-l), k<l \leq L-1$. Also, the first $L$ elements of block $i$ is $v$. Therefore, $\hat{s}_{i}(k-l)=v(k-l), l=0,1, \cdots, k$. Hence

$$
\begin{aligned}
x_{i}(k) & =\sum_{l=0}^{k} h_{i}(l) v(k-l)+\sum_{l=k+1}^{L-1} h_{i}(l) v(L+k-l) \\
& =\sum_{l=0}^{L-1} h_{i}(l) v\left(\langle k-l\rangle_{L}\right)+\eta_{i}(k) .
\end{aligned}
$$

The FFT can be used to estimate the channel. Let $V(k)$, $\hat{H}_{i}(k), \Upsilon_{i}(k)$ and $\hat{X}_{i}(k)(k=0,1, \cdots, L-1)$ be the length- $L$ DFT of $v(n), h_{i}(n), \eta_{i}(n)$ and $x_{i}(n)(n=0,1, \cdots, L-1)$, respectively, then

$$
\hat{X}_{i}(k)=V(k) \hat{H}_{i}(k)+\Upsilon_{i}(k), \quad k=0,1, \cdots, L-1 .
$$

Ignoring the noise, we get $\hat{H}_{i}(k)=\hat{X}_{i}(k) / V(k)$. Thus, the channel is estimated as

$$
\hat{h}_{i}=\operatorname{IDFT}\left(\hat{H}_{i}\right)
$$

with inverse discrete Fourier transform (IDFT). Since $V(k)$ is independent of the symbols, it can be computed once for all and stored. Therefore, only two FFTs of length $L$ are needed for the channel estimation. The computational complexity is $\mathrm{O}\left(L \log _{2} L\right)$ operations (multiplications and additions).

In order to minimize the impact of noise, we need to choose the CP carefully. Let $\mathbf{V}=\operatorname{diag}(V)$. Then, from (2), $\hat{H}_{i}=$ $\mathbf{V}^{-1} \hat{X}_{i}-\mathbf{V}^{-1} \Upsilon_{i}$. Therefore, the best CP should be the one such that $\mathrm{E}\left(\left\|\mathbf{V}^{-1} \Upsilon_{i}\right\|^{2}\right)$ is minimized with the constraint of fixed power for the $\mathrm{CP}$, where $\mathrm{E}(y)$ means the mathematical expectation of a random variable $y$. Note that

$$
\begin{aligned}
\mathrm{E}\left(\left\|\mathbf{V}^{-1} \Upsilon_{i}\right\|^{2}\right) & =\operatorname{tr}\left(\mathrm{E}\left(\mathbf{V}^{-1} \Upsilon_{i}\left(\mathbf{V}^{-1} \Upsilon_{i}\right)^{\dagger}\right)\right) \\
& =\operatorname{tr}\left(\mathbf{V}^{-1} \mathrm{E}\left(\Upsilon_{i} \Upsilon_{i}^{\dagger}\right) \mathbf{V}^{-\dagger}\right)
\end{aligned}
$$

If the noise is white, then $\mathrm{E}\left(\Upsilon_{i} \Upsilon_{i}^{\dagger}\right)=L \sigma_{n}^{2} \mathbf{I}$, where $\sigma_{n}^{2}$ is the variance of the noise. So, the best $\mathrm{CP}$ should minimize

$$
\frac{1}{|V(0)|^{2}}+\cdots+\frac{1}{|V(L-1)|^{2}}
$$

at the constraint of $|v(0)|^{2}+\cdots+|v(L-1)|^{2}=c$, where $c$ is the power of the CP. Since $V$ is the DFT of $v,\|V\|^{2}=L\|v\|^{2}$. Thus, the constraint is equivalent to $|V(0)|^{2}+\cdots+|V(L-1)|^{2}=L c$. It is easy to prove that the ideal pilot is the one satisfying

$$
|V(0)|=\cdots=|V(L-1)| .
$$

However, this condition cannot always be met if the CP symbols are chosen from a finite alphabet. In general, we can search all possible CPs to get the best one (minimize (4)). However, the search for a length- $L$ best $\mathrm{CP}$ is time-consuming when $L$ is large, especially when the size of the constellation is large. In practice, we can search the best CP for BPSK constellation, which is relatively easy to achieve, and then use the $\mathrm{CP}$ for all QAM constellation.

\section{Symbol ReCoverabiLITY AND EQUALIZATION}

After the channel is estimated, equalization can be done in the same way as in the conventional single carrier system. Thanks to the cyclic prefix, $x_{i}(L: M-1)$ is the cyclic convolution of $h_{i}(l)$ and $\bar{s}_{i}(n)$. By zero padding the channel $h_{i}(l)$ into length $N$, and denoting the length- $N$ DFTs of $x_{i}(L: M-1), \eta_{i}(L: M-1)$, $\bar{s}_{i}(n)$, and $h_{i}(l)$ by $X_{i}(k), \Gamma_{i}(k), \bar{S}_{i}(k)$, and $H_{i}(k)$ respectively, then

$$
X_{i}(k)=\bar{S}_{i}(k) H_{i}(k)+\Gamma_{i}(k), \quad k=0,1, \cdots, N-1 .
$$

The notations used here should not be confused with those in (2). Two commonly used equalization methods, namely, the zeroforcing (ZF) and linear minimum mean-square error (MMSE), can be constructed as follows.

\section{ZF equalizer:}

$$
\bar{s}_{i}=\left(\begin{array}{c}
s_{i} \\
v
\end{array}\right)=\operatorname{IDFT}\left(\frac{X_{i}}{H_{i}}\right) .
$$

MMSE equalizer (assume the noise is white and its variance is known):

$$
\bar{s}_{i}\left(\begin{array}{c}
s_{i} \\
v
\end{array}\right)=\operatorname{IDFT}\left(\frac{H_{i}^{*} \odot X_{i}}{H_{i} \odot H_{i}^{*}+\frac{\sigma_{n}^{2}}{\sigma_{s}^{2}}}\right),
$$

where $\sigma_{s}^{2}$ is the variance of the transmitted symbol, and $\odot$ is the elementwise multiplication operation. 
When some of the $H_{i}(k)$ are zeros, the symbols cannot be recovered in conventional CP-SC or OFDM systems, which is a major problem in CP-SC or OFDM. However, thanks to the known block $v$ in each CP-SC block, symbols are recoverable in the proposed structure even if some $H_{i}(k)$ are zeros.

Let $\mathbf{D}_{i}=\operatorname{diag}\left(H_{i}\right)$ (Matlab notation) and $\mathbf{W}$ be the length- $N$ DFT transform matrix. Partition the matrix into $\mathbf{W}=\left(\mathbf{W}_{1} \mathbf{W}_{2}\right)$, where $\mathbf{W}_{1}$ and $\mathbf{W}_{2}$ have $N_{1}$ and $L$ columns, respectively. It follows from (6) that

Therefore

$$
\mathbf{D}_{i}\left(\mathbf{W}_{1} \mathbf{W}_{2}\right)\left(\begin{array}{c}
s_{i} \\
v
\end{array}\right)=X_{i}-\Gamma_{i}
$$

$$
\mathbf{D}_{i} \mathbf{W}_{1} s_{i}=X_{i}-\mathbf{D}_{i} \mathbf{W}_{2} v-\Gamma_{i} \stackrel{\text { def }}{=} \bar{X}_{i}-\Gamma_{i} .
$$

Let $\mathbf{B}_{i}=\mathbf{D}_{i} \mathbf{W}_{1}$, whose size is $N \times N_{1}$. It is obvious that $s_{i}$ is recoverable from (10) if and only if $\mathbf{B}_{i}$ is of full rank. From the special structure of the DFT matrix, it is easy to verify that arbitrary $N_{1}$ rows of $\mathbf{W}_{1}$ are linear independent. Thus, if the number of zeros in $H_{i}(k)$ is not more than $L$, the matrix $\mathbf{B}_{i}$ will be of full rank. Furthermore, since the channel order is not greater than $L-1$, it has at most $L-1$ zeros, that is, $H_{i}(k)$ should never have more than $L$ zeros. Hence, we obtain the following very important theorem.

Theorem 2: In the absence of noise, input symbols $s_{i}$ are always recoverable from (10) regardless of the channel null locations.

From (10), a least square (LS) estimation of the symbols is

$$
s_{i}=\left(\mathbf{W}_{1}^{\dagger} \mathbf{D}_{i}^{\dagger} \mathbf{D}_{i} \mathbf{W}_{1}\right)^{-1} \mathbf{W}_{1}^{\dagger} \mathbf{D}_{i}^{\dagger} \bar{X}_{i} .
$$

We call this method CP-aided zero forcing (CPZF). If the noise is white and its variance is known, a CP-aided MMSE (CPMMSE) estimation can be constructed as

$$
s_{i}=\mathbf{W}_{1}^{\dagger} \mathbf{D}_{i}^{\dagger}\left(\mathbf{D}_{i} \mathbf{W}_{1} \mathbf{W}_{1}^{\dagger} \mathbf{D}_{i}^{\dagger}+\frac{N \sigma_{n}^{2}}{\sigma_{s}^{2}}\right)^{-1} \bar{X}_{i}
$$

A major problem in these two methods is the high computational complexity, because matrix inverting is required. To avoid this problem, iterative methods can be used for solving (10). For example, based on the conjugate gradient method [10], an iterative method is constructed to find the $s_{i}$ such that $\| \mathbf{D}_{i} \mathbf{W}_{1} s_{i}-$ $\bar{X}_{i} \|^{2}$ minimized as follows.

$$
\begin{aligned}
& \text { Given an initial value for } s_{i} ; \\
& r=\mathbf{W}_{1}^{\dagger} \mathbf{D}_{i}^{\dagger}\left(\bar{X}_{i}-\mathbf{D}_{i} \mathbf{W}_{1} s_{i}\right) ; \\
& \rho_{0}=\|r\|^{2} ; \\
& \text { for } k=1: K \\
& \quad \text { if } k==1 ; p=r ; \\
& \quad \text { else } \beta=\rho_{k-1} / \rho_{k-2} ; p=p+\beta r ; \\
& \quad \text { end } \\
& \quad \omega=\mathbf{W}_{1}^{\dagger} \mathbf{D}_{i}^{\dagger} \mathbf{D}_{i} \mathbf{W}_{1} p ; \alpha=\rho_{k-1} /\left(p^{\dagger} \omega\right) ; \\
& \quad s_{i}=s_{i}+\alpha p ; r=r-\alpha \omega ; \rho_{k}=\|r\|^{2} ; \\
& \text { end }
\end{aligned}
$$

where $K$ is the number of iterations. The initial value for $s_{i}$ can be obtained by the ZF or MMSE method described in (7) and (8), respectively. This method is called CP-aided iterative zero

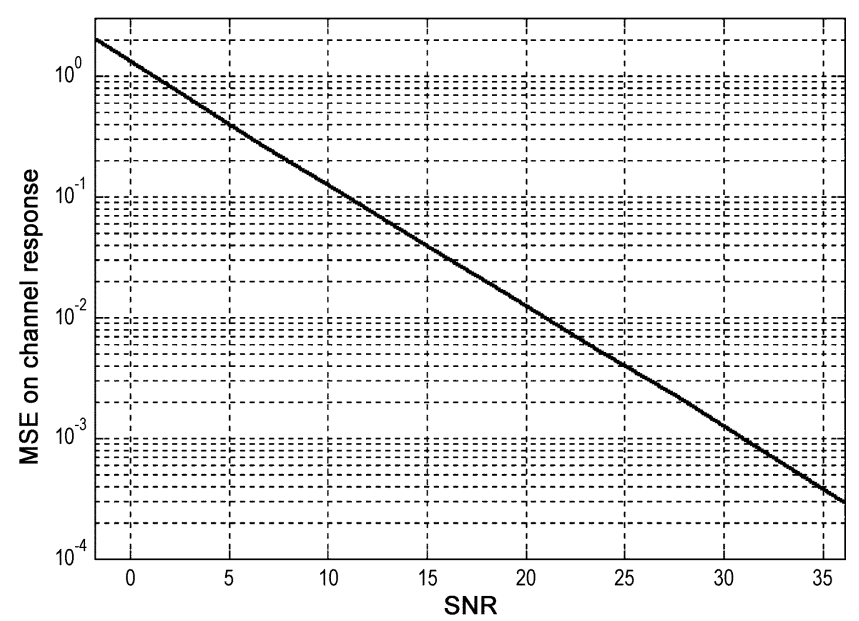

Fig. 2. Channel MSE versus SNR.

forcing (CPIZF). The multiplication of the matrix $\mathbf{W}_{1}$ or $\mathbf{W}_{1}^{\dagger}$ with a vector can be realized by FFT to reduce the computational complexity. In fact, $\mathbf{W}_{1} y=\mathbf{W}\left(\begin{array}{l}y \\ 0\end{array}\right)$, and $\mathbf{W}_{1}^{\dagger} z$ is only part of the vector $\mathbf{W}^{\dagger} z$.

\section{SimULATIONS}

In the following, signal-noise-ratio (SNR) means the ratio of the average received signal power with the average noise power as SNR $=\left(\mathrm{E}\left(\left|x_{i}(k)-\eta_{i}(k)\right|^{2}\right)\right) / \mathrm{E}\left(\left|\eta_{i}(k)\right|^{2}\right)$. The MSE between the estimated and true channel response is defined as $\operatorname{MSE}=\left(\sum_{l=0}^{L-1}|h(l)-\hat{h}(l)|^{2}\right) /\left(\sum_{l=0}^{L-1}|h(l)|^{2}\right)$, where $h(l)$ and $\hat{h}(l)$ are the true and estimated channel responses respectively.

For BPSK, there are 16 length- 8 pilot CPs and 96 length- 16 pilot CPs which minimize (4). An example is $(1,1,-1,1,-1$, $-1,-1,-1)$ (for length 8 ) and $(1,-1,-1,1,-1,-1,1,1,1$, $-1,-1,-1,1,-1,-1,-1$ ) (for length 16). Note that the DC component of any optimal pilot $\mathrm{CP}$ must be nonzero. In the simulations, we choose $L=16, N=128$ and use the length-16 best pilot CP for BPSK given above for all QAM constellations. Uncoded system is considered. The CP has the same average power as that of the symbols. The discrete channels are generated randomly and vary from block to block (a new random channel is generated for a new block). Fig. 2 shows the MSE of the estimated channel versus SNR, where the MSE is averaged over 100 Monte Carlo realizations (for each realization, a random channel is generated and random symbols are transmitted). It is seen that the channel estimation method is effective. Comparisons on bit error rate (BER) for ZF, CPZF, CPIZF, MMSE, and CPMMSE equalizations are conducted, where the channels are estimated by the proposed method. All results on BER are obtained by averaging over 4000 Monte-Carlo realizations. Since the test is based on finite number of samples, very small $\left(<10^{-5}\right)$ BER is meaningless and therefore it is not shown in the figures. The comparison on BER for ZF and CPZF equalizations is given in Fig. 3, where lines with marks $\circ, \triangle$, $\nabla$, and $\square$ are for constellation of BPSK, 4-QAM, 16-QAM, and 64-QAM, respectively. This figure shows that CPZF is substantially better than the ZF and supports the statement of symbol recoverability in Theorem 2 . In the 4000 different channels, quite 


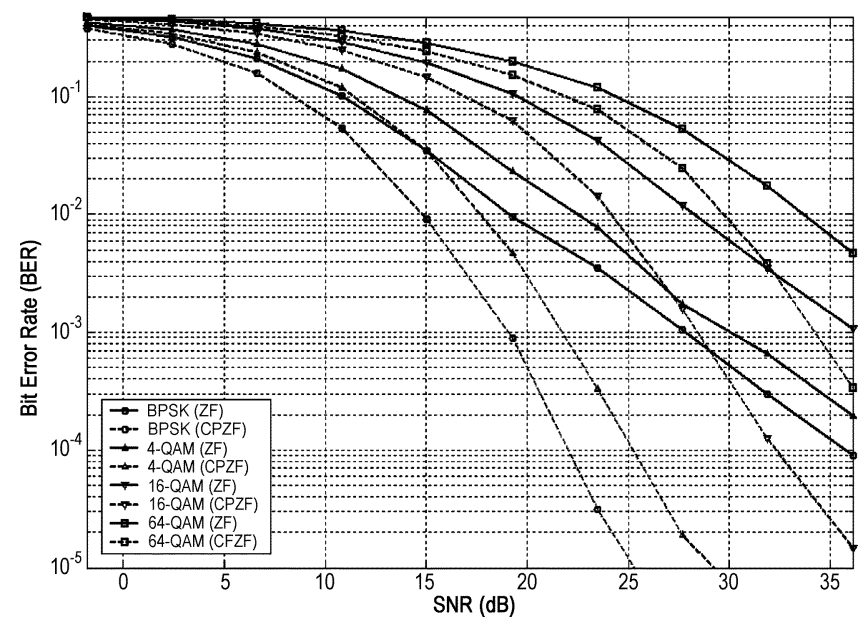

Fig. 3. Comparison of BER for ZF and CPZF.

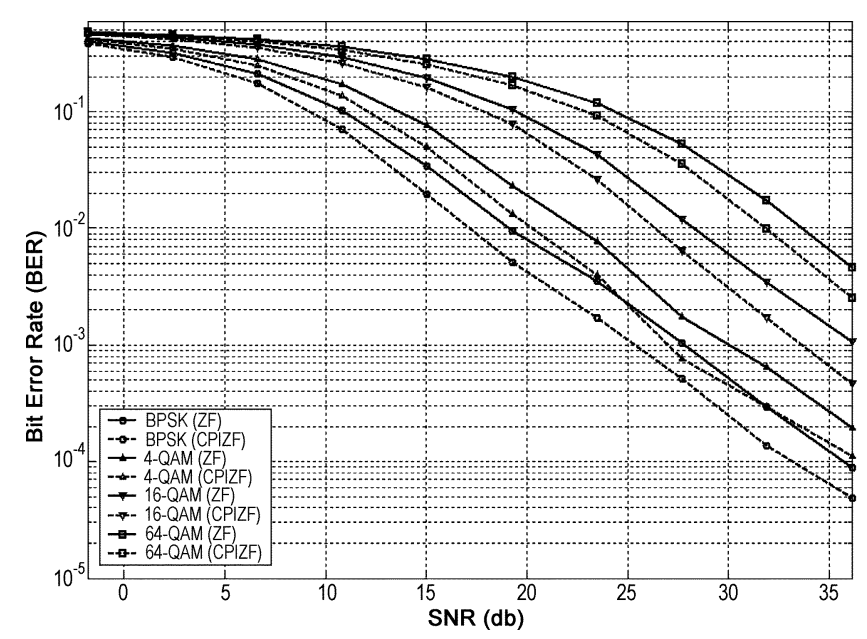

Fig. 4. Comparison of BER for ZF and CPIZF.

a few of them are "bad" (some DFT coefficients of the channel are nearly zeros) and therefore the ZF cannot recover the symbols correctly at those cases but the CPZF can. Fig. 4 gives the comparison for ZF and CPIZF, where the initial value for CPIZF is obtained from the result of $\mathrm{ZF}$, and the number of iteration is 10. It shows that CPIZF is always better than $\mathrm{ZF}$ and worse than CPZF. Based on the theory of conjugate gradient method [10], if the number of iterations becomes 112 (the order of matrix $\left.\mathbf{B}_{i}^{\dagger} \mathbf{B}_{i}\right)$, CPIZF will converge to CPZF. The ZF, CPZF, and CPIZF do not need any information on the statistics of noise and symbol. Fig. 5 shows the comparison on BER for MMSE and CPMMSE methods, where the noise is assumed to be white and exact noise and signal variances are used. In a fast-variant environment, the noise variance may also varies from time to time and therefore it is virtually impossible to get its exact value. Moreover, the noise may not be white. So, this figure can only be viewed as an ideal case.

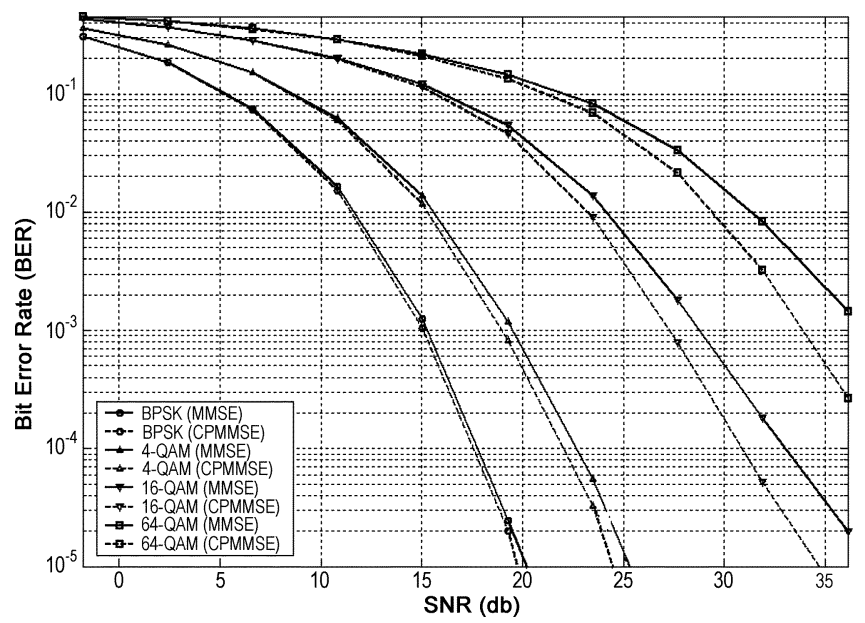

Fig. 5. Comparison of BER for MMSE and CPMMSE.

\section{CONCLUSION}

We have proposed a structure named PCP-SC. It has been proved that the structure offers channel estimation by FFT for fast fading channel and ensures input symbols recoverable regardless of the channel null locations. Furthermore, the known symbols in the suffix and CP can also be used for synchronization. The expense is the slight decrease in data rate.

\section{REFERENCES}

[1] H. Sari, G. Karam, and I. Jeanclaude, "Transmission techniques for digital terrestrial TV broadcasting," IEEE Commun. Mag., vol. 33, no. 2, pp. 100-109, Feb. 1995.

[2] L. Deneire, B. Gyselinckx, and M. Engels, "Training sequence versus cyclic prefix-A new look on single carrier communication," IEEE Commun. Lett., vol. 5, no. 7, pp. 292-294, Jul. 2001.

[3] D. Falconer, S. L. Ariyavisitakul, A. Benyamin-Seeyar, and B. Eidson, "Frequency domain equalization for single-carrier broadband wireless systems," IEEE Commun. Mag., vol. 40, no. 4, pp. 58-66, Apr. 2002.

[4] N. Benvenuto and S. Tomasin, "On the comparison between OFDM and single carrier modulation with a DFE using a frequency-domain feedforward filter," IEEE Trans. Commun., vol. 50, no. 6, pp. 947-955, June 2002.

[5] J. Louveaux, L. Vandendorpe, and T. Sartenaer, "Cyclic prefixed single carrier and multicarrier transmission: bit rate comparison," IEEE Commun. Lett., vol. 7, no. 4, pp. 180-182, 2003.

[6] C. Li and S. Roy, "Subspace based blind channel estimation for OFDM by exploiting virtual carriers," IEEE Trans. Wireless Commun., vol. 2, no. 1, pp. 141-145, 2003.

[7] B. Muquet, M. D. Courville, and P. Duhamel, "Subspace-based blind and semi-blind channel estimation for OFDM systems," IEEE Trans. Signal Processing, vol. 50, no. 7, pp. 1699-1712, Jul. 2002.

[8] X. Wang and K. J. Liu, "Adaptive channel estimation using cyclic prefix in multicarrier modulation system," IEEE Commun. Lett., vol. 3, no. 10, pp. 291-293, Oct. 1999.

[9] S. Zhou and G. B. Giannakis, "Finite-alphabet based channel estimation for OFDM and related multicarrier systems," IEEE Trans. Commun., vol. 49, no. 8, pp. 1402-1414, Aug. 2001.

[10] C. T. Kelley, Iterative Methods for Linear and Nonlinear Equations. Philadelphia, PA: SIAM, 1995. 